\title{
Biometrics Authentication of Aerial Handwritten Signature Using a Convolutional Neural Network
}

\author{
Kohei Nakanishi*, Shin-ichi Ito*, Momoyo Ito+ and Minoru Fukumi+ \\ Tokushima University \\ 2-1, Minami-Josanjima, Tokushima, 770-8506, Japan \\ *\{c501737039, s.ito $\} @$ tokushima-u.ac.jp \\ $+\{$ momoito, fukumi $\} @$ is.tokushima-u.ac.jp
}

\begin{abstract}
Recent years, biometrics authentication is receiving attention by development of information society. In this paper, we propose a personal authentication system, which uses behavior characteristics among biometrics. We focus on aerial handwritten signature, because it is difficult to forge it, and there is no risk of loss. In this paper, we measure signatures using Leap Motion Controller. It can measure three dimensional space coordinates with high accuracy. We divide signature data into three axial directions of coordinates $\mathrm{XYZ}$ in order to use them as one-dimensional data. We carry out preprocessing to signature data and normalize them. Next, we use deep learning based on a convolutional neural network for feature extraction and identification. In this experiment, we prepare data obtained from six subjects. We obtain genuine data of one subject. The remaining five subjects are used to create forgery data. We classify signature data into two classes. We conduct deep learning in which convolutional neural network carries out 10,000 cycles learning in one trial. We carry out this trial 5 times and evaluate mean accuracy by cross validation for two types of genuine data. The average discrimination accuracy of this experiment are $97.0 \%$ and $95.9 \%$. In addition, the false rejection rates are $9.6 \%$ and $19.2 \%$. The false acceptance rate are $0.8 \%$ and $0.1 \%$.
\end{abstract}

Keywords: Biometrics, Human Interface, CNN, Leap Motion, Signature.

\section{Introduction}

Recent years, password and IC cards are often used to security systems. These are the systems based on

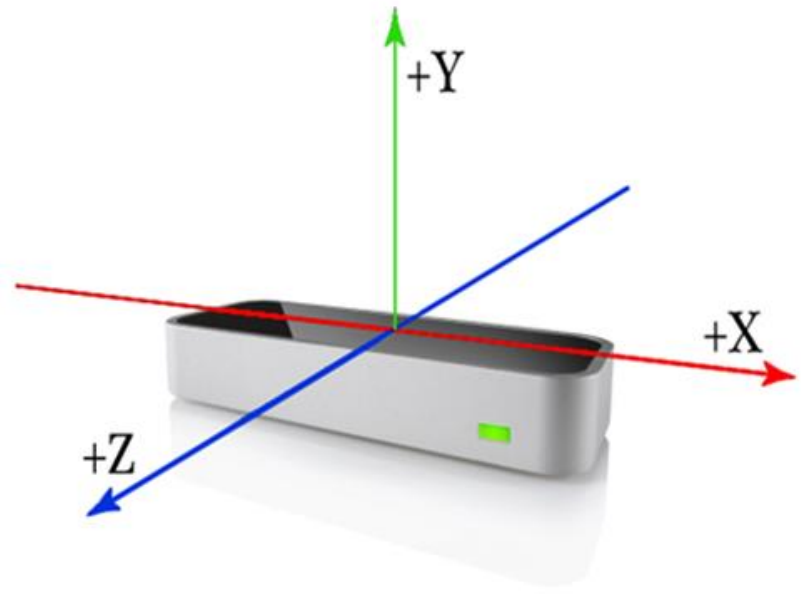

Fig. 1. Leap motion controller.

knowledge and objects. It is easy to introduce them into many kinds of security systems. However, there are some drawbacks such as forgetting, theft and forgery. In this paper, we focus on aerial handwritten signature as a method of biometrics authentication. This means to write signatures in the air. After that, we obtain habits and features from that motion, and authenticate it by these features. The aerial handwritten signature belongs to behavioral characteristics in biometrics. The behavioral characteristics has reproducible and universal properties. Its authentication accuracy is lower than that by physiological characteristics. However, we are accustomed to sign many years. Therefore, signatures have enough reproducible and universal properties of signature motion. In addition, aerial handwritten signature does not leave its trace. Thus, it would be difficult to forge aerial handwritten signature. If another person counterfeits a signature, we can change registration signature in free. The physiological characteristics does not have this advantage. In aerial handwritten signature, it is necessary to sign the same 


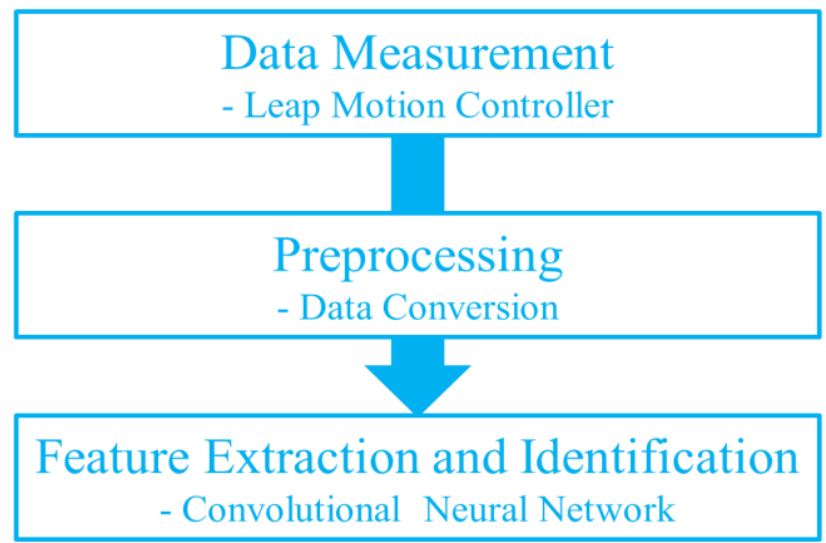

Fig. 2. Flow of the proposed method.

trajectory in the same behavior. They are used for both of the habit included in a motion and signature characteristics. From the above, aerial handwritten signature has excellent properties of biometrics. Therefore, in this paper we propose a personal authentication by the aerial handwritten signature.

\section{Related Work}

There have been few biometrics authentication by leap motion device (Fig 1). In a related work, accuracy of "Leap Signature Recognition using Hoof and Hot Features"(1) is $91 \%$. The accuracy of "Sign Language Recognition using Leap Motion Controller" (2) is $82 \%$, and the accuracy of "A Leap Password based Verification System"(3) is $81 \%$. On the one hand, the accuracy of general fingerprint authentication systems is $99 \%$, and the accuracy of face authentication systems is $97 \%$. As mentioned above, the accuracy of personal authentication system by Leap Motion device is relatively low compared to that of other authentication systems. Therefore, we proposed a new authentication method of aerial handwritten signature using a convolutional neural network.

\section{Proposed Method}

We propose a method of personal authentication by aerial handwritten signature. The proposed method in this paper is composed of three steps shown in Fig.2. We measure aerial signatures with the Leap Motion Controller. Then, the signature data are preprocessed for input to deep learning. We use deep learning by a convolutional neural network for feature extraction and identification.
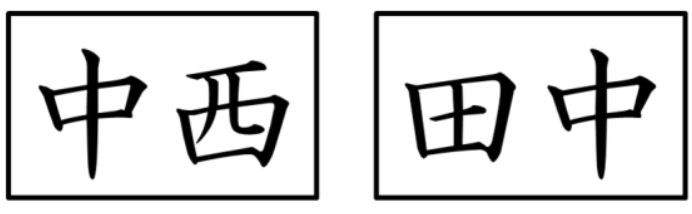

Fig. 3 Genuine signature "Nakanishi" and "Tanaka".

\subsection{Measurement of aerial handwritten signature}

We measure aerial handwritten signatures with the Leap Motion Controller, which is a small USB peripheral device. It has the following characteristics. First, motions of hands and fingers are measured in three dimensional space coordinates. Second, measurement resolution is $0.01 \mathrm{~mm}$. Finally, we do not contact to a monitor and a mouse. The hands or fingers are measured above the sensor. The coordinates are shown in Fig1. In this paper, we obtain two kind of signature data (Fig 3). These are Japanese kanji.

\subsection{Pre-processing}

In this paper, we divide signature data into three axial directions of coordinates XYZ (Fig 4). We treat signature data as one-dimensional data. This is done in order to capture finger motion in each axis. We then apply three processing to the signature data such as trimming, conversion and equalization.

\subsubsection{Trimming}

In this processing, we remove parts before the starting point and after the ending point of each signature data. There are useless parts before and after writing data in the signature data. The coordinate values of those parts hardly vary. Those have no meaning as data. Therefore, they are deleted by threshold processing. Specifically, in the XY plane, $10 \mathrm{~mm}$ data at the beginning and the end are removed.

\subsubsection{Conversion}

In this processing, we convert coordinate information into motion information. Coordinate data is also different even in the same person by a different starting point. Therefore, we calculate the difference between successive coordinate data as follows:

$$
x_{t}^{\prime}=x_{t}-x_{t-1}
$$

where parameters " $\mathrm{x}$ ", and " $\mathrm{t}$ " are a value of the $\mathrm{X}$-axis, and 

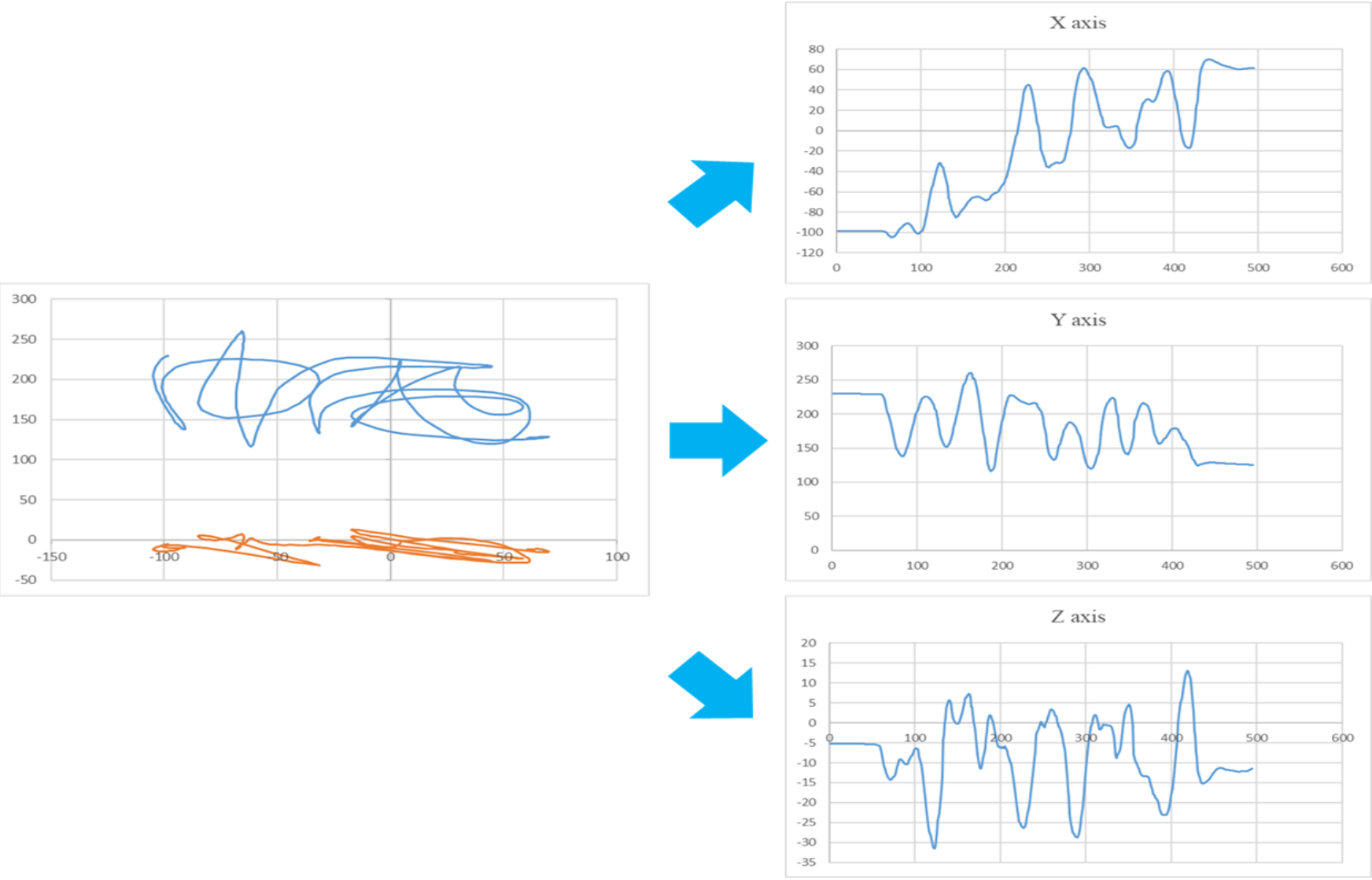

Fig. 4. Signature is divided into $\mathrm{XYZ}$ axis directions.

a time, respectively. The same processing is carried out in Y-axis and the Z-axis. As a result, we can obtain the motion information of fingers at each time.

\subsubsection{Equalization}

In this processing, we equalize data length. The measured signatures have different length. Therefore, it is necessary to equalize all data length for input to a convolutional neural network. In this paper, we equalize the data length by a linear interpolation method. There are two kinds of data in this paper. Their data length are 350 and 250. Fig 5 shows graphs after three preprocessing.

\section{Experiment}

In this section, we show descriptions of experiments by the proposed method. We prepare data obtained from six subjects. We obtain genuine data of one subject. The remaining five subjects data are used to create forgery data.

\subsection{Signature Measurement}

In this experiment, we measure signatures by tracking the coordinates of a finger using the Leap Motion device. The frame rate of measurement is $60 \mathrm{fps}$. The attitude of the subject's signature is free, and the subjects sit on a chair in a relaxed way. The aerial handwritten signature is unfamiliar to many people. The subjects sufficiently practice in advance. We indicate the trajectory of a two dimensional plane by the index finger on a PC display by a software in signature measurement (Fig 6). We give a visual feedback for the subjects. In this experiment, the subjects sign their last names, which are familiar with writing for many years.

\subsubsection{Genuine data measurement}

The feedback is not carried out at the time of actual measurement. Because, subjects may have consciousness on the screen and cannot concentrate on movement.

\subsubsection{Forgery data measurement}

As in the case of genuine, the feedback is not carried out at the time of actual measurement. When making a 

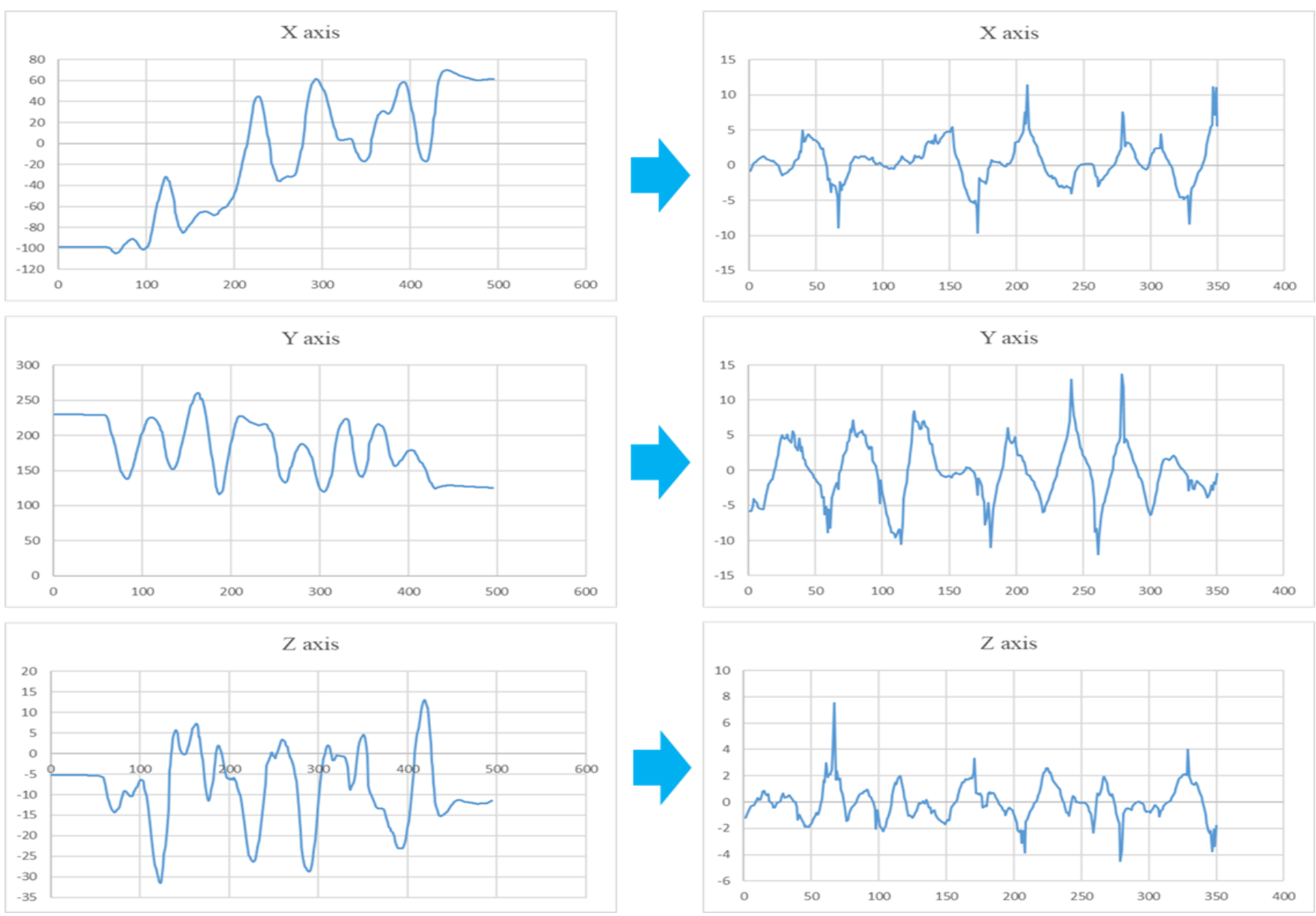

Fig. 5. After three preprocessing.

forgery, we do not present the movement of the genuine handwriting.

\subsection{Signature to use}

We measured each signature 90 times per subject, and obtained 540 data. We measured genuine data for 15 data per day for a total of 3 days. Therefore, the total of genuine data per person is 45 data. In this study, two persons of the subjects create data of genuine. We obtain 90 data of genuine. We measured forgery data for 15 data per day for a total of 3 days. The total of forgery data for per person is 45 data. In this study, we prepared two types of genuine data. Therefore, the forgery data per person is 90 data. We prepared five subjects, and then the total of forgery data is 450. We divided signature data into three for cross validation (Table 1). We use 180 data (30 data / person) for learning and 90 data ( 15 data / person) for test.

\subsection{Network Structure}

We divide signature data into XYZ axial directions by the preprocessing. The data length is normalized to 350 or 250 per each axis. Fig 7 shows the structure of a convolutional neural network used in the experiments. In this paper, we implement deep learning using TensorFlow by Google. We give data of each XYZ axis into the input layer. We represent data for each axis as one feature map. We set movement width of the filters to one at the convolution layers. At the sixth layer, we classify signature data into two classes. If an output value exceeds an authentication threshold value set in advance, the input data belongs to the class, which corresponds to the unit. When we carry out convolution operations, we unify feature map size by zero padding. Furthermore, we implement a drop out to prevent over training. Thereby, we can expect to improve accuracy. In this paper, we use 40 percent rate of units to drop out learning. We use a rectified linear unit in the second to fifth layers. We use a softmax function in the sixth layer. 


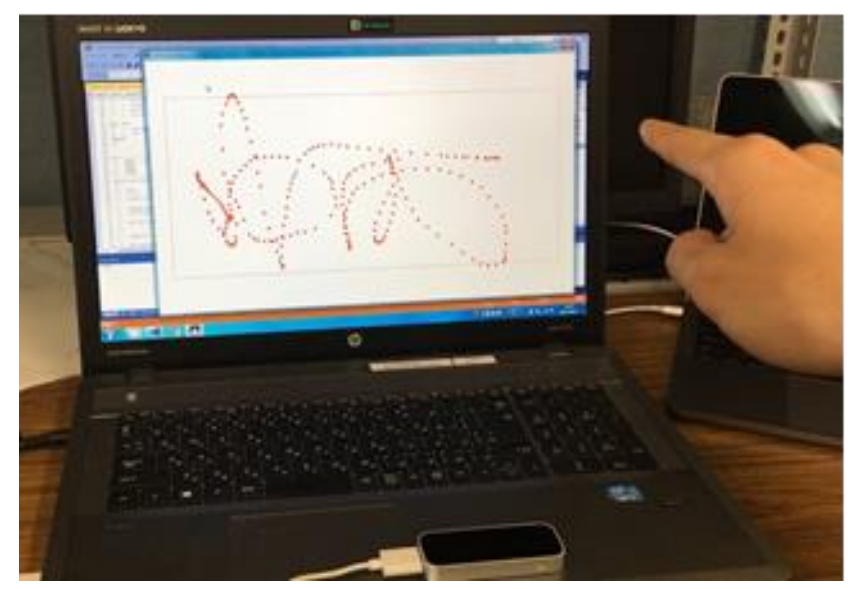

Fig. 7. Screen of software for signature measurement.

Table 1. Signature to use.

\begin{tabular}{|c|c|c|c|}
\hline \multirow{2}{*}{$\begin{array}{c}\text { Learning } \\
\text { Data }\end{array}$} & $\begin{array}{c}\text { Data } \\
\text { Set }\end{array}$ & Genuine & Forgery \\
\cline { 2 - 4 } & 1 & 30 data & 30 data x 5 \\
\cline { 2 - 4 } & 3 & 30 data & 30 data x 5 \\
\hline \multirow{2}{*}{$\begin{array}{c}\text { Test } \\
\text { Data }\end{array}$} & 2 & 15 data & 15 data $\times$ data 5 \\
\cline { 2 - 4 } & 1 & 15 data $\times 5$ \\
\cline { 2 - 4 } & 3 & 15 data & 15 data x 5 \\
\hline
\end{tabular}

\section{Results and Consideration}

In experiments, we conduct deep leaning in which $\mathrm{CNN}$ carries out 10,000 cycles learning in one trial. We conduct this trial 5 times for each data set and calculate the average. Table 2 shows experimental results. FRR means "false rejection rate", and FAR means "false acceptance rate". We carried out personal authentication of signature by multi classification in our previous paper ${ }^{(4)}$. In our previous paper, the accuracy is $98.0 \%$, the FRR is $4.8 \%$, and the FAR is $0 \%$. When the present results are compared with the previous results, FRR has got worse than them. In the

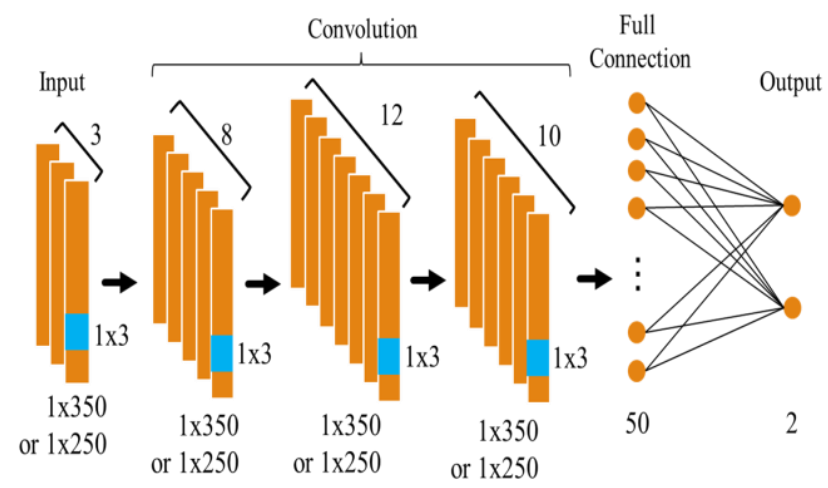

Fig. 6. Network structure.

Table 2. Experimental results.

\begin{tabular}{|c|c|c|c|c|c|}
\hline 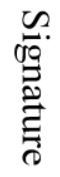 & & $\begin{array}{c}\text { Data } \\
1\end{array}$ & $\begin{array}{c}\text { Data } \\
2\end{array}$ & $\begin{array}{c}\text { Data } \\
3\end{array}$ & Average \\
\hline \multirow{3}{*}{ 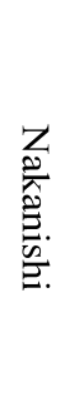 } & Accuracy & $98.8 \%$ & $95.5 \%$ & $96.9 \%$ & $97.0 \%$ \\
\hline & FRR & $2.2 \%$ & $20 \%$ & $6.6 \%$ & $9.6 \%$ \\
\hline & FAR & $0.8 \%$ & $0 \%$ & $1.76 \%$ & $0.8 \%$ \\
\hline \multirow{3}{*}{ 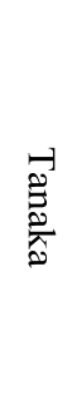 } & Accuracy & $93.6 \%$ & $96.9 \%$ & $97.3 \%$ & $95.9 \%$ \\
\hline & FRR & $31.1 \%$ & $11.1 \%$ & $15.5 \%$ & $19.2 \%$ \\
\hline & FAR & $0 \%$ & $0.4 \%$ & $0 \%$ & $0.1 \%$ \\
\hline
\end{tabular}

previous experiment, subjects signed while looking at the trajectory of the index finger on a PC display. However, we do not give visual feedback in this experiment. This means that we want subjects to concentrate to aerial signature motion. In this paper, there are no feedback. Therefore, we think reproducibility of signature declined. As shown in Table 2, we think that the result of the data set 1 of the signature "Tanaka" is particularly bad. In this paper, five subjects per person create forgery data. FAR are $0.8 \%$ and 

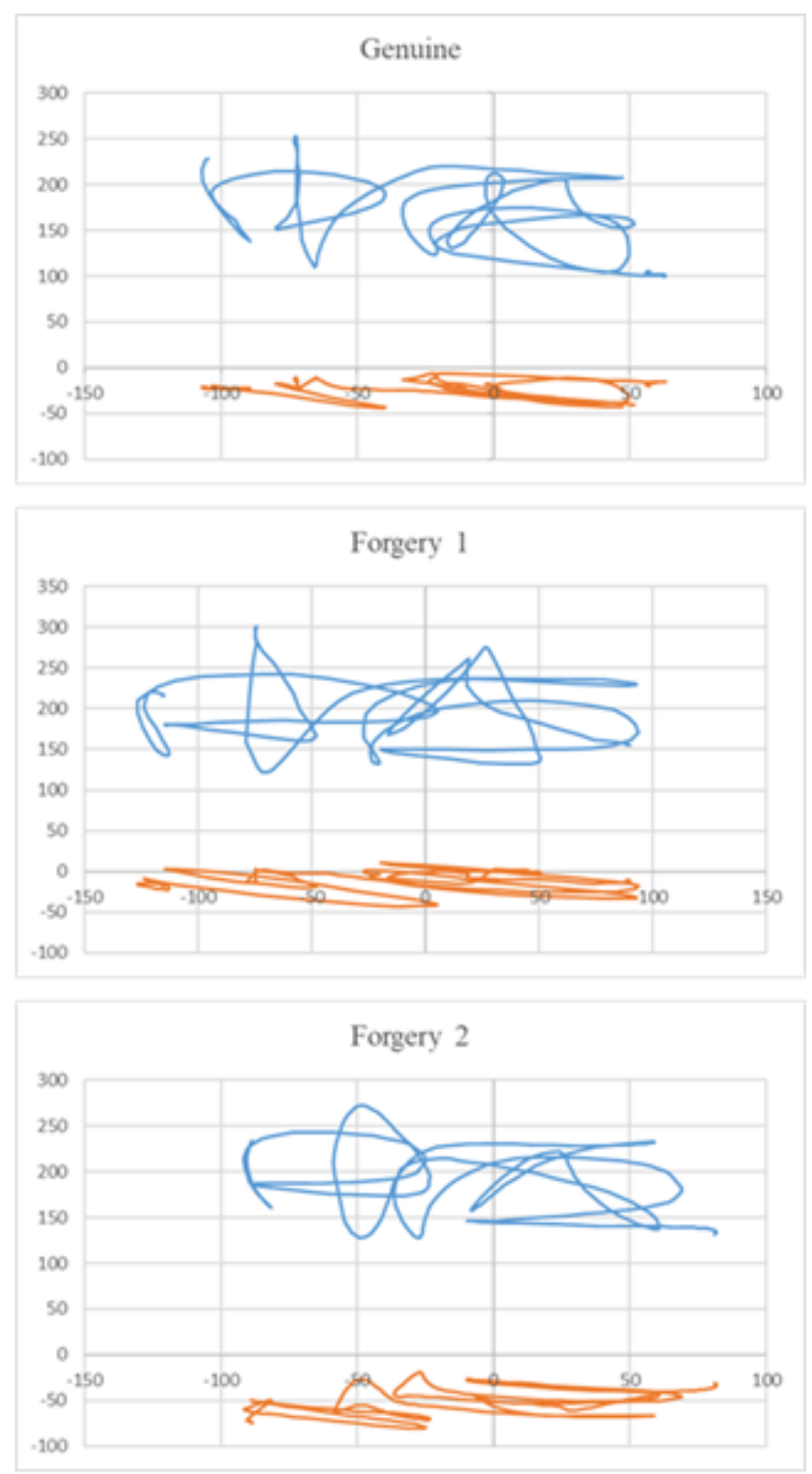

Fig. 8. Differences in stroke order.

$0.1 \%$, which are good results. We think it is difficult to forge without presenting the movement of genuine handwriting. For example, signature stroke order is significantly different for each subject. Signatures for different subjects look alike (Fig.8). However, the movements are very different. Therefore, we think that it is a main factor where we can identify forgery. In future plan, we present to the movement of genuine handwriting to subjects. By doing so, we can evaluate accuracy in a more severe situation.

\section{Conclusion}

In this paper, we focused on aerial handwritten signature for biometrics personal authentication. We measured signatures using the Leap Motion Controller. We obtained two kind of signature data. These are Japanese kanji. We transformed coordinate information of the signature to motion information by preprocessing. Then, we used deep learning based on a convolutional neural network for feature extraction and identification. We classified signature data into two classes. We used the learning data which were genuine writing data actually measured. In this paper, we implemented deep learning by TensorFlow. We can change the layer structure by the use of TensorFlow easily. The accuracy in two genuine signature were $97.0 \%$ and $95.9 \%$. In addition, FRR were $9.6 \%$ and $19.2 \%$. FAR were $0.8 \%$ and $0.1 \%$. We would like to increase accuracy by increasing signature data and improving signature environment.

\section{References}

(1) Ishan Nigam, Mayank Vatsa, and Richa Singh : "Leap Signature Recognition Using Hoof and Hot Features", IEEE International Conference on Image Processing (ICIP), Oct. 2014.

(2) Makiko Funasaka, Yu Ishikawa, Masami Takata, and Kazuki Joe : "Sign Language Recognition using Leap Motion Controller", International Conference Par and Dist Proc Tech and Appl, 13 December 2015

(3) Aman Chahar, Shivangi Yadav, Ishan Nigam, Richa Singh, Mayank Vatsa, : "A Leap Password based Verification System", IEEE International Conference on Biometric Theory, Applications and Systems (BTAS), Sept.2015

(4) Kohei Nakanishi, Daiki Hiraoka, Shin-ichi Ito, Momoyo Ito and Minoru Fukumi : "Biometrics Authentication of Aerial Handwritten Signature Using a Convolutional Neural Network", 4th International Conference, ICAT'Rome, Rome, Italy, November 23-25, 2016 